\title{
Nonlinear eigenvalue problems
}

\section{L.Ju. Fradkin and G.C. Wake}

The object of this paper is to prove two new results on the nature of the spectrum of a class of nonlinear elliptic eigenvalue problems. In the first case, sufficient conditions are given for which the spectrum is bounded and, in the second case, conditions are given for which the spectrum is open.

In this paper we consider a nonlinear boundary value problem of the form

$$
\begin{aligned}
L u=\lambda F(u), & \text { in } \Omega, \\
B_{\varepsilon} u=0, & \text { on } \partial \Omega,
\end{aligned}
$$

where $\Omega$ is a bounded domain of $\mathrm{R}^{n}(n \geq 2)$ with boundary $\partial \Omega$ of class $c^{2+\alpha}, 0<\alpha<1$, and closure $\bar{\Omega}$. The operator $L$ is a real selfadjoint, second-order, uniformly elliptic differential operator defined by

$$
(L u)(x) \equiv-\sum_{i, k=1}^{n} \frac{\partial}{\partial x_{i}}\left(a_{i j}(x) \frac{\partial u}{\partial x_{k}}(x)\right)+a(x) u(x),
$$

where $a_{i k}=a_{k i}$, and $\sum_{i, k=1}^{n} a_{i k}(x) p_{i} p_{k} \geq \gamma_{0} \sum_{i=1}^{n} p_{i}^{2}>0$, if $x \in \Omega$, $p \in R^{n}$.

We assume that the functions $a_{i k}, a, \frac{\partial}{\partial x_{i}} a_{i k}$, for all $i, k$, are in the class $c^{\alpha}(\bar{\Omega})$ and $a(x) \geq 0$ for all $x \in \Omega$.

Let $\frac{\partial u}{\partial v}$ be the conormal derivative defined by

Received 28 February 1975. 


$$
\frac{\partial u}{\partial v} \equiv \sum_{i, k=1}^{n} m_{i} a_{i k} \frac{\partial u}{\partial x_{k}},
$$

where $\left(m_{1}, \ldots, m_{n}\right)$ is the unit outward normal at $x \in \partial \Omega$. Then ${ }_{\varepsilon}$ is a first order boundary operator defined as follows:

$$
B_{\varepsilon}(u)=a_{0}(x) u+\varepsilon \frac{\partial u}{\partial v}
$$

where $\varepsilon$ is either 0 or $1, a_{0}(x) \in c^{2-\varepsilon+\alpha}(\partial \Omega)$, and

$$
\begin{aligned}
& a_{0}(x)>0, \text { if } \varepsilon=0, \\
& a_{0}(x) \geq 0, \text { if } \varepsilon=1 .
\end{aligned}
$$

Moreover, we require that $a(x)$ and $a_{0}(x)$ are not both identically zero. We assume that $L$ is self-adjoint with boundary operator $B_{\varepsilon}$.

Let $S$ be an arbitrary subset of $R$. For any real-valued function $g(x, u(x))$ we denote by the corresponding capital letter the Nemytskii operator $G(u)$, that is, if $g: \bar{\Omega} \times S \rightarrow R$, then for every $u: \bar{\Omega} \rightarrow S$, $G(u)$ is defined by $G(u)(x) \equiv g(x, u(x))$. We will suppose that $f(x, u(x)) \equiv F(u)(x)$ in (1) satisfies some or all of the following conditions:

H.I $\left|f\left(x_{1}, u_{1}\right)-f\left(x_{2}, u_{2}\right)\right| \leq \gamma(s)\left(\left|x_{1}-x_{2}\right|^{\alpha}+\left|u_{1}-u_{2}\right|^{\alpha}\right)$, for a.l $\left(x_{i}, u_{i}\right) \in \bar{\Omega} \times S, i=1,2$, where $S=[0, N]$ and $N>0$;

H.2 $f(x, 0) \geq 0$, for all $x \in \bar{\Omega}$ (but it is not identically zero);

H. $3 f\left(x, u_{1}(x)\right)>f\left(x, u_{2}(x)\right)$, if $u_{1}(x)>u_{2}(x)$;

H.3' $f_{u} \in C(\bar{\Omega} \times S)$, where $f_{u}(x, u(x)) \equiv F_{u}(u)(x)$ is the Fréchet derivative; $f_{u}>0$, for all $(x, u) \in \bar{\Omega} \times S ; S \subseteq \mathrm{R}^{+}$;

H.4 $\inf _{(x, u) \in \bar{\Omega} \times \mathrm{R}^{+}} f_{u}(x, u) \equiv c>0$.

Our assumptions imply a maximum principle for the operators $\left(L, B_{\varepsilon}\right)$, if $u \in C^{2}(\Omega) \cap C^{\varepsilon}(\bar{\Omega})$, and allow us to apply two known theorems.

THEOREM A. If $f \in C^{\alpha}(\bar{\Omega})$, the linear boundary value problem 


$$
\begin{gathered}
L u=f, \quad x \in \Omega, \\
B_{\varepsilon} u=0, \quad x \in \partial \Omega,
\end{gathered}
$$

has a unique solution $u \in c^{2+\alpha}(\bar{\Omega})$. (For proof of this, see Amann [1], Ladyzhenskaya and Ural'tseva [5].)

THEOREM B. If $r(x)>0, r(x) \in C(\bar{\Omega})$, the boundary value problem

$$
\begin{array}{ll}
L v=\mu r(x) v, & \text { in } \Omega, \\
B_{\varepsilon} v=0, & \text { on } \partial \Omega,
\end{array}
$$

has a principal eigenvalue $\mu_{1}>0$ and positive eigenfunction $v \in C^{\perp}(\bar{\Omega}) \cap C(\bar{\Omega})$. (For proof of this, see Keller [3] or Keller and Cohen [4].)

We will be interested in the positive solutions $u$ of (1) for which $u \in C^{2+\alpha}(\bar{\Omega})$. The set $\Lambda$ of the corresponding positive $\lambda$ will be called the spectrum. We will study questions connected with the critical point $\lambda^{*}=\sup \Lambda$ and will use the following theorems for problem (1).

THEOREM C. If H.I, 2, 3 hold and $\lambda^{\prime}$ is a point of the spectrum then all $\lambda \in\left(0, \lambda^{\prime}\right]$ are points of the spectrum. For all such $\lambda$ there exists a minimal solution $u(\lambda, x) \in C^{2+\alpha}(\bar{\Omega}) ;$ moreover $u(\lambda, x)$ is an increasing function of $\lambda$. (For proof of this, see Keller [3] or Keller and Cohen [4].)

THEOREM D. If H.1, 2, 3 hold and $f$ is bounded, then $\lambda^{*}=\infty$. (For proof of this, see Keller [3] or Keller and Cohen [4].)

Using the theorems above, we can provide two further results concerning the value of $\lambda^{*}$. The first of these gives some further conditions, different from those in Theorem $D$, under which $\lambda^{*}$ is finite. The second theorem gives criteria under which $\lambda^{*} \vDash \Lambda$.

THEOREM 1. If $f$ satisfies H.1, 2, 3', 4 and problem (1) has some spectrum, then $\lambda^{*}$ is a finite number.

Proof. For such an $f$ it is known (Keller and Cohen, [4], Theorem 4.1) that

$$
\lambda \leq \mu_{1}(\lambda),
$$


where $\mu_{1}(\lambda)$ is the principal eigenvalue of the problem,

$$
\begin{aligned}
L v & =\mu f_{u}(x, u(\lambda, x)) v, \\
B_{\varepsilon} v & =0 .
\end{aligned}
$$

The function $u(\lambda, x) \in c^{2+\alpha}(\bar{\Omega})$ and H. 3' holds, hence $f(x, u(\lambda, x)) \in C(\bar{\Omega})$ and $\mu_{1}(\lambda), v(x)$ exist by Theorem B. The variational characterization of $\mu_{1}(\lambda)$ is as follows:

$$
\mu_{1}(\lambda)=\min _{\psi \in \Psi} \frac{J(\psi)}{\left(\psi, f_{u} \psi\right)}
$$

where $f_{u} \equiv f_{u}(x, u(\lambda, x))$,

$$
J(\psi)=\int_{\Omega}\left(\sum_{i, k=1}^{n} a_{i k}(x) \frac{\partial \psi}{\partial x_{i}} \frac{\partial \psi}{\partial x_{k}}+a(x) \psi^{2}\right) d x+\int_{\partial \Omega} a_{0}(x) \psi^{2} d \theta(x)
$$

and the set of admissible functions is

$$
\Psi=\left\{\psi \mid \psi(x)>0, \psi(x) \in C^{\mathcal{L}}(\Omega) \cap C(\bar{\Omega}), \psi(x)=0 \text { on } \partial \Omega_{1}\right\},
$$

where $\partial \Omega_{1}$ is the part of $\partial \Omega$ for which $\varepsilon=0$. If $\psi_{0} \in \Psi$, then using H. 4 and (4) we obtain:

$$
\mu_{1}(\lambda) \leq \frac{J\left(\psi_{0}\right)}{\left(\psi_{0}, f_{u} \psi_{0}\right)} \leq \frac{J\left(\psi_{0}\right)}{c\left(\psi_{0}, \psi_{0}\right)}<\infty \text {, for all } \lambda \text { in } \Lambda .
$$

It follows from (2) and (5) that $\lambda^{*}$ is a finite number. //

NOTE. H. 4 holds for example for any convex $f$, that is, $f_{u}$ increasing with $u$.

There is another interesting question concerning $\lambda^{*}$. This is the question as to whether the spectrum is open, that is, does $\lambda^{*} \in \Lambda$ ? To answer this question, we need to introduce two further restrictions on $f$, which are:

H. 5 there exist $p, q \in C^{\alpha}(\bar{\Omega})$ such that $f(x, u) \leq p(x)+q(x) u$, for all $(x, u) \in \Omega \times \mathrm{R}^{+}$;

H.6 for any $N_{1}>0$, there exists $N_{2}>N_{1}$, such that 


$$
f_{u}\left(x, N_{2}\right)<f_{u}\left(x, N_{1}\right), x \in \Omega
$$

We are now able to generalise the result in Keller and Cohen [4, Corollary 4.1.2] concerning this question.

THEOREM 2. If H.1, 2, 3', 4, 5, 6 are satisfied, then $\lambda^{*} \equiv \Lambda$.

Proof. By Theorem 1, $\lambda^{*}$ exists under the assumptions H.1, 2, 3', 4. To prove our theorem by contradiction, we assume that $\lambda^{*} \epsilon \Lambda$, that is, there exists $u\left(\lambda^{*}, x\right)$, for all $x \in \Omega$. As H.5 holds, we can use Keller and Cohen [4, Corollary 3.3.3] to conclude that, for any $N_{2}>\mathrm{u}\left(\lambda^{*}, x\right)$,

$$
\mu_{1}\left(f_{u}\left(x, N_{2}\right)\right) \leq \lambda^{*}
$$

where $\mu_{1}\left(f_{u}\left(x, N_{2}\right)\right)$ is the principal eigenvalue of the problem in Theorem B, with $r(x)=f_{u}\left(x, N_{2}\right)$. By H.6, we can choose $N_{2}$ so that

$$
f_{u}\left(x, N_{2}\right)<f_{u}\left(x, u\left(\lambda^{*}, x\right)\right) .
$$

Since $\mu_{1}$ is monotonic with the weight function, we get

$$
\mu_{1}\left(f_{u}\left(x, N_{2}\right)\right)>\mu_{1}\left(f_{u}\left(x, \mathrm{u}\left(\lambda^{*}, x\right)\right)\right) \text {. }
$$

The same argument as in Amann [2, Theorem 3.4], which in fact applies for all $f$ satisfying H.1, 2, 3', not just asymptotically linear $\dot{f}$ (as in Amann), shows that

$$
\mu_{1}\left(f_{u}\left(x, u\left(\lambda^{*}, x\right)\right)\right)=\lambda^{*}
$$

Equations (8) and (9) taken together give us a contradiction with equation (6). $/ /$

\section{References}

[1] Herbert Amann, "On the existence of positive solutions of nonlinear elliptic boundary value problems", Indicana Univ. Math. J. 21 (1971), 125-146.

[2] Herbert Amann, "Positive solutions of convex nonlinear eigenvalue problems", Indiana Univ. Math. J. (to appear). 
[3] H.B. Keller, "Some positone problems suggested by nonlinear heat generation", Bifurcation theory and nonlinear eigenvalue problems, 217-255 (Benjamin, New York, Amsterdam, 1969).

[4] Herbert B. Keller and Donald S. Cohen, "Some positone problems suggested by nonlinear heat generation", J. Math. Mech. 16 (1967), 1361-1376.

[5] Olga A. Ladyzhenskaya and Nina N. Ural'tseva, Linear and quasilinear elliptic equations (Mathematics in Science and Engineering, 46. Translated by Scripta Technica. Academic Press, New York and London, 1968).

Department of Mathematics,

Victoria University of Wellington,

Well ington,

New Zealand. 